\title{
MicroRNA-146 function in the innate immune transcriptome response of zebrafish embryos to Salmonella typhimurium infection
}

\author{
Anita Ordas ${ }^{1,2 \dagger}$, Zakia Kanwal ${ }^{1 \dagger}$, Valesca Lindenberg ${ }^{1}$, Julien Rougeot ${ }^{1}$, Matyas Mink ${ }^{2}$, Herman P Spaink ${ }^{1}$
} and Annemarie H Meijer ${ }^{1 *}$

\begin{abstract}
Background: MicroRNAs (miRNAs) have recently been shown to play important roles in development of the immune system and in fine-tuning of immune responses. Human miR-146 family members are known as inflammation-inducible miRNAs involved in negative feedback regulation of Toll-like receptor (TLR) signalling. Dysregulation of the miR-146 family has often been linked to inflammatory diseases and malignancies. This study reports on miR-146a and miR-146b as infection-inducible miRNAs in zebrafish, which has emerged as a model species for human disease.
\end{abstract}

Results: Using a custom-designed microarray platform for miRNA expression we found that both members of the zebrafish miR-146 family, miR-146a and miR-146b, were commonly induced by infection of zebrafish embryos with Salmonella typhimurium and by infection of adult fish with Mycobacterium marinum. The induction of these miRNAs was confirmed by Taqman miRNA assays. Subsequently, we used zebrafish embryos, in which adaptive immunity is not yet active, as an in vivo system to investigate the role of miR-146 in the innate immune response to S. typhimurium infection. Knockdown of traf6 and use of myd88 mutants demonstrated that the induction of miR-146a and miR-146b by S. typhimurium infection was affected by disruption of the MyD88-Traf6 pathway that mediates transduction of TLR signals and cytokine responses. In turn, knockdown of miR-146 itself had no major effects on the expression of known targets of MyD88-Traf6 signalling. Instead, RNA sequencing analysis showed that miR-146 knockdown led to an increased induction of six members of the apolipoprotein gene family in S. typhimurium-infected embryos.

Conclusion: Based on microarray analysis and Taqman miRNA assays we conclude that members of the miR-146 family, which is highly conserved between fish and human, are induced by bacterial infection in zebrafish in a MyD88 and Traf6 dependent manner. The combined knockdown of miR-146a and miR-146b in zebrafish embryos infected with S. typhimurium had no major effect on the expression of pro-inflammatory genes and transcription factors known to be downstream of the MyD88-Traf6 pathway. In contrast, apolipoprotein-mediated lipid transport emerged as an infection-inducible pathway under miR-146 knockdown conditions, suggesting a possible function of miR-146 in regulating lipid metabolism during inflammation.

Keywords: MicroRNA, miR-146, Innate immunity, Infection, Salmonella typhimurium, Mycobacterium marinum, MyD88, Traf6, Apolipoproteins, Zebrafish

\footnotetext{
* Correspondence: a.h.meijer@biology.leidenuniv.nl

${ }^{\dagger}$ Equal contributors

'Institute of Biology, Leiden University, Einsteinweg 55, 2333 CC Leiden, The Netherlands

Full list of author information is available at the end of the article
} 


\section{Background}

Timely activation as well as termination of inflammatory responses is essential for proper functioning of the immune system. A balanced output of the vertebrate immune response is dependent on several regulatory mechanisms, in which microRNAs (miRNAs) have recently emerged as new players with critical importance [1]. Several human diseases, including cancer, autoimmune diseases, and chronic inflammatory disorders, have been associated with dysregulation of miRNA expression both in a positive or negative regulatory manner [1-5]. MiRNAs are evolutionary conserved, genome-encoded small RNAs ( 22 nucleotides) that down-regulate gene expression at the post-transcriptional level by either translational repression or by mRNA degradation through binding to the $3^{\prime}$-UTR of their target mRNAs [6]. MiRNAs were found to have roles in a diverse range of processes ranging from development, cellular differentiation, hematopoiesis, apoptosis, and growth, to the functioning of the immune system [7-10].

The role of host miRNAs in bacterial infections has been addressed in several previous studies. MiRNAs were observed to be differentially regulated by Toll-like receptor (TLR)-mediated recognition of bacterial molecules. For instance, lipopolysaccharide (LPS) stimulation of TLR4 and downstream NFkB activity induced miR-146, miR-147, and miR-155 [11-13]. Mice deficient in miR-155 could not be protected by vaccination against Salmonella typhimurium infection and showed strong defects in T-cell cytokine production [14]. In another study, Salmonella typhimurium infection was found to induce rapid down-regulation of let-7 miRNA family members in macrophages, thereby leading to an upregulation of let-7 targets, the IL- 6 and IL-10 cytokines [15]. IL-10 production in LPS-stimulated macrophages was also found to be regulated by miR-98 [16]. Furthermore, miR-29 was found to suppress immune responses to Listeria monocytogenes and Mycobacterium tuberculosis by targeting IFN- $\gamma$ [17].

The LPS-inducible miR-146 family comprises two members in human, miR-146a and miR-146b, which are transcribed from different genes on chromosome 5 and 10 , respectively [18]. They have the same seed sequence (i.e. the sequence essential for the binding of the miRNA to its mRNA target) and differ in their mature sequence by only two nucleotides at the 3 'end. Dysregulation of miR-146a and miR-146b has been observed in many types of malignant tumors and several studies implicate these miRNAs as metastasis suppressors [18-23]. MiR-146a has also been implicated in different autoimmune diseases, including systemic lupus erythematosus, rheumatoid arthritis and Sjögren's syndrome $[24,25]$. The first indication of the role of miR-146a/b in innate immunity came from work of Taganov et al. [11], showing increased expression of these miRNAs in the human monocytic THP-1 cell line when triggered by
LPS. Promoter analysis revealed that induction of the miR-146a gene by LPS, TNF $\alpha$, and IL-1 is mediated by the NFKB transcription factor [11]. In addition, 3 '-UTR luciferase reporter assays demonstrated that the TLR signalling intermediates IRAK1 and TRAF6 are potential targets of miR-146a and miR-146b [11]. These data suggested that the miR-146 miRNAs function in a negative feedback pathway of TLR and cytokine signalling by targeting IRAK1 and TRAF6 mRNAs for downregulation, a conclusion supported by recent analysis of miR-146a knockout mice [26]. MiR-146a has also been shown to function as a negative regulator of interferon (IFN) signalling by targeting the IRF5 and STAT-1 transcription factors [27] and to control the resolution of T-cell responses in mice [28].

The zebrafish provides a useful model to study innate immunity, which is the primary line of defence against infections during the first few weeks of development, when functional adaptive immunity is not yet present $[29,30]$. The zebrafish model combines an efficient genetic toolbox with excellent possibilities for high resolution imaging of host-microbe interactions at the early life stages (embryos and larvae), when zebrafish are transparent $[29,30]$. Many zebrafish infection models for bacterial pathogens have recently been developed, among which the S. typhimurium and M. marinum models are the best characterized [29]. The major signalling pathways of the innate immune system are conserved within all vertebrates [31] and the repertoire of zebrafish miRNAs is well described [32,33]. Since miRNAs are strongly conserved among all vertebrates, the advantages of the zebrafish model organism may be exploited to elucidate miRNA functions in the vertebrate host response to bacterial infections. As in mammals, the zebrafish miR-146 family has two members, named dre-miR-146a and dremiR-146b, which are present within genes located on chromosome 13 and 21, respectively. The IRAK1 and TRAF6 homologs of both zebrafish and human contain putative target sites for miR-146 in their 3'-UTRs, suggesting that miR-146 feedback control of TLR signalling is evolutionary conserved. Here we report on a microarray study of miRNA expression, which showed that miR-146a and miR-146b are commonly induced by infection of zebrafish embryos with Salmonella typhimurium and by infection of adult fish with Mycobacterium marinum. We demonstrate the requirement of the MyD88-Traf6 pathway for the infection-triggered induction of miR-146a/b in the zebrafish embryo model. Furthermore, we used morpholino knockdown to suppress the function of miR-146a and miR-146b and analyzed the effects of this down-regulation by RNA deep sequencing (RNAseq) of embryos infected with Salmonella typhimurium. While no major effects on known targets of the MyD88-Traf6 pathway were observed, apolipoprotein- 
mediated lipid transport emerged as a novel infectioninducible pathway under control of miR-146a/b.

\section{Results}

Microarray analysis identifies infection-inducible miRNAs in zebrafish embryos and adults

In order to study the effects of bacterial infection on miRNA expression profiles in zebrafish we examined two infection conditions that are known from previous mRNA transcriptome studies to elicit a strong proinflammatory immune response: embryos at 8 hours post infection (hpi) with the S. typhimurium SL1027 strain and adult fish that were in the end stage of disease at 6 days post infection (dpi) with the M. marinum Mma20 strain [34,35]. Since embryos rely solely on innate immunity and adults also have adaptive immunity, the combination of these infection models allows determining the contribution of the two arms of the immune system to the induction of miRNAs [29,30]. To quantify microRNA gene expression profiles we used a custom designed $8 \times 15$ k Agilent zebrafish array. S. typhimurium infection of one-day old embryos resulted in differential expression of probes for 15 miRNAs annotated in miRBase (8 up-regulated, 7 down-regulated), while the M. marinum-infected adults showed differential expression of probes for 57 miRNAs (37 up-regulated, 20 down-regulated) (Figure 1A,B). The miRNA platform also contains probes for predicted hairpin structures in the zebrafish genome that might cover additional miRNAs. A large number of these probes showed differentially expression in the adult infection study (235 upregulated, 687 down-regulated), while a much lower number was affected by infection of embryos (27 upregulated, 37 down-regulated). Since the biological relevance of these predicted small RNAs is currently unknown, we did not consider them further in this study, but focused on the known miRNAs annotated in miRBase. Several of these miRNAs were commonly upregulated by both of the infection conditions, including miR-21 (mature miRNA and its star sequence), miR-29a, miR-29b, miR-146a, and miR-146b (Figure 1A). Since zebrafish embryos have not yet developed adaptive immunity, it can be concluded that the context of innate immunity is sufficient to induce the expression of these miRNAs.

miR-146a and miR-146b are induced during zebrafish infection with $S$. typhimurium and $M$. marinum

MiRNAs of the miR-146 family, which emerged as infection-inducible miRNAs from the microarray analysis of embryonic and adult zebrafish, have previously been linked to the innate immune response in mammalian systems [11]. To confirm the induction of these miR-146 family members we analyzed miR-146a and
miR-146b expression by TaqMan qPCR analysis. MiR222 , which showed unaltered expression in the microarray study, was used as a control for normalization. In agreement with the microarray data, miR-146a and b were specifically induced in embryos at 8 hours post injection (hpi) with the S. typhimurium SL1027 strain (Figure 2A). Their induction was also observed in infections with an attenuated LPS mutant (Ra) strain, SF1592 (Figure 2B). Furthermore, induction of miR-146a and miR-146b was detected in zebrafish larvae at an advanced stage of $M$. marinum Mma20 infection (Figure 2C). The increase of miR-146a expression in embryos infected with $S$. typhimurium could be completely blocked with a morpholino targeting this miRNA (Figure 2A,B) and this morpholino was effective even up to the larval stage in reducing $M$. marinum-induced miR-146a expression (Figure 2C). The S. typhimuriuminduction of miR-146b in embryos could also be reduced by morpholino treatment, (Figure 2A,B), but the miR146b morpholino was no longer effective at the larval stage where $M$. marinum infection was analyzed. In agreement with the microarray data, induction of miR$146 \mathrm{a}$ and miR-146b was also confirmed in adult zebrafish infected with M. marinum (Figure 2D).

\section{Infection-inducible expression of miR-146a and miR-146b} is affected by defects in signalling via the MyD88-Traf6 pathway

We used the S. typhimurium embryo infection model to investigate the dependency of miR-146a and miR-146b induction on TLR pathway genes. First, we used a previously described morpholino knockdown model for traf6, a central intermediate in TLR and TNF receptor signalling [36]. The S. typhimurium-induced expression levels of miR-146a and miR-146b were significantly lower in traf6 knockdown embryos compared to controls (Figure 3A). Next, we analyzed miR-146a and miR-146b induction in a myd88 mutant line [37]. Similar as under traf6 knockdown conditions, miR-146a and miR-146b were still infection-inducible in myd88 mutant embryos, but their expression levels were significantly higher in infected wild type siblings (Figure 3B). Therefore, we conclude that miR-146a and miR-146b induction is at least partially dependent on MyD88 and Traf6.

\section{MiR-146a and miR-146b knockdown does not affect leukocyte development in zebrafish embryos}

Loss of function studies in mice and zebrafish suggested a possible role of miR-146a in the development of myeloid cells, in addition to its proposed inhibitory effect on proinflammatory signalling [38]. To investigate the possible requirement of miR-146a and miR-146b for leukocyte development in zebrafish embryos, we designed two different morpholinos for each miRNA (Additional file 1: Figure S1). 


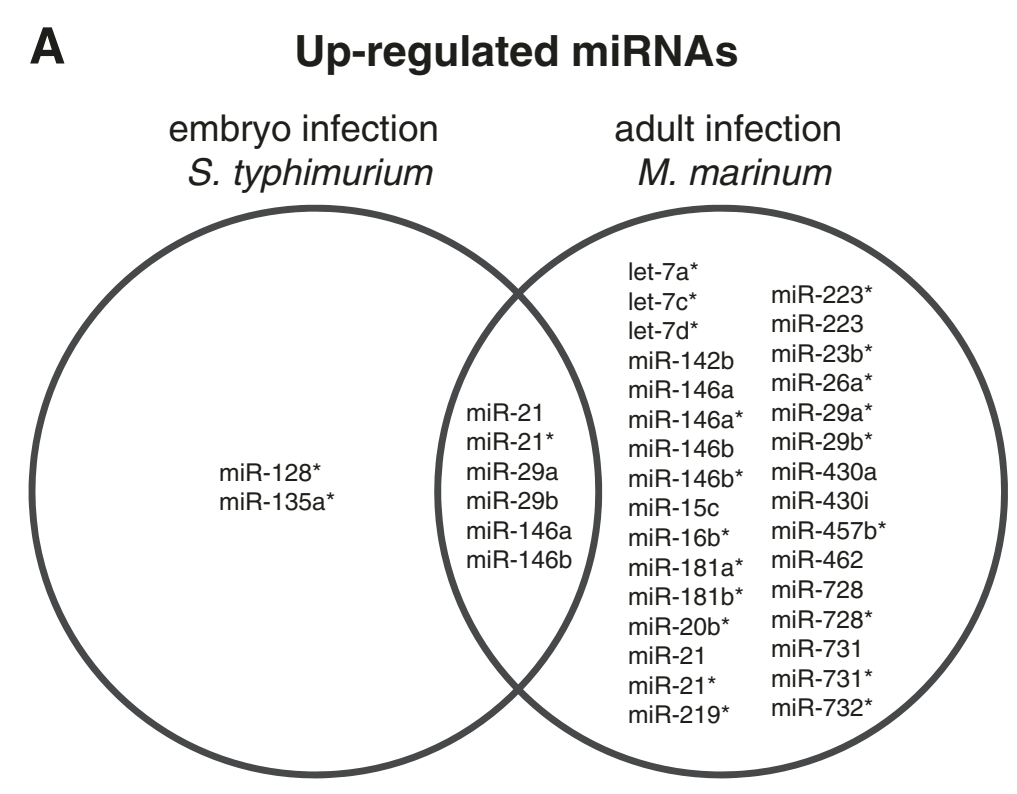

\section{B Down-regulated miRNAs}

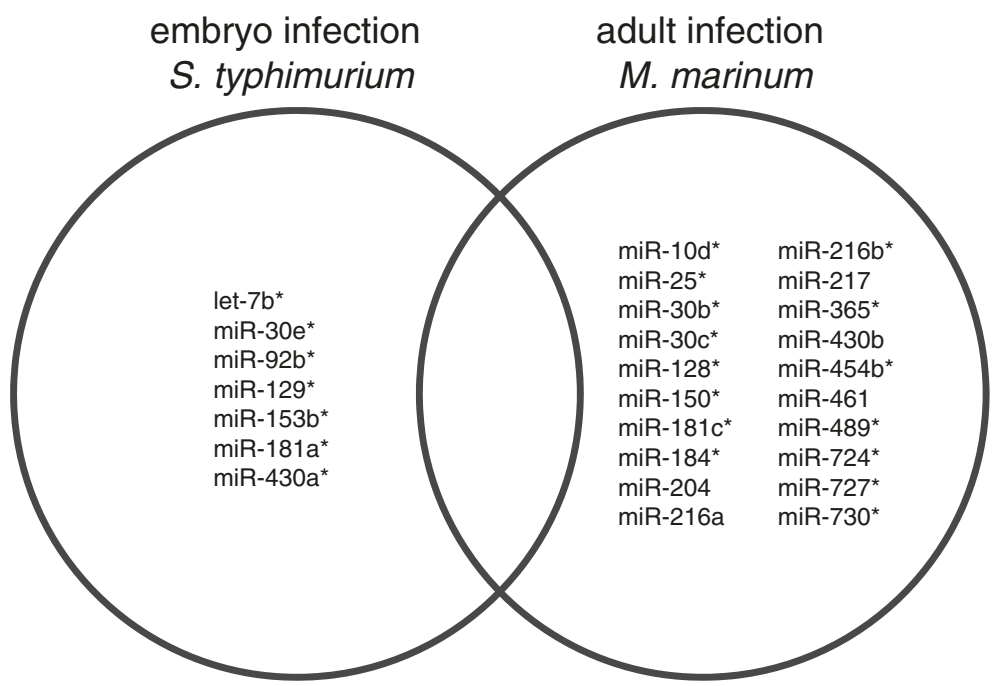

Figure 1 Microarray analysis of miRNA expression during bacterial infections of zebrafish embryos and adult fish. The Venn diagrams show the miRBase annotated miRNAs or miRNA star sequences $\left(^{*}\right)$ that were up-regulated $(\mathbf{A})$ or down-regulated $(\mathbf{B})$ during infection of zebrafish embryos with S. typhimurium SL1027 or infection of adult zebrafish with M. marinum Mma20. S. typhimurium infection of embryos was performed by micro-injection into the caudal vein at $28 \mathrm{hpf}$ and miRNA expression was analyzed at $8 \mathrm{hpi}$ in comparison with control embryos mock-

injected with PBS. Adult zebrafish were infected with M. marinum by intraperitoneal infection and miRNA expression at 6 dpi was compared with PBS-injected controls.

The efficiency of the knockdown was confirmed by TaqMan qPCR analysis, showing that basal expression of miR-146a and miR-146b (Figure 4A,B) as well as their infection inducibility (Figure 2A-C) was reduced by morpholino treatments. Both of the morpholinos designed for miR-146a (146aMO1 and 146aMO2) did not affect miR-146b expression, therefore showing specific knockdown of miR-146a only (Figure 4A,B). However, one of the
miR-146b morpholinos (146bMO1) showed knockdown of both miR-146a and miR146b expression (Figure 4A,B). To assess leukocyte numbers we performed immunostaining for the pan-leukocytic marker L-plastin and histochemical staining for myeloperoxidase (Mpx) activity, a specific enzyme of neutrophils. First, we analyzed the effect of combined knockdown of miR-146a and miR-146b at $2 \mathrm{dpf}$. No differences were observed between controls and 


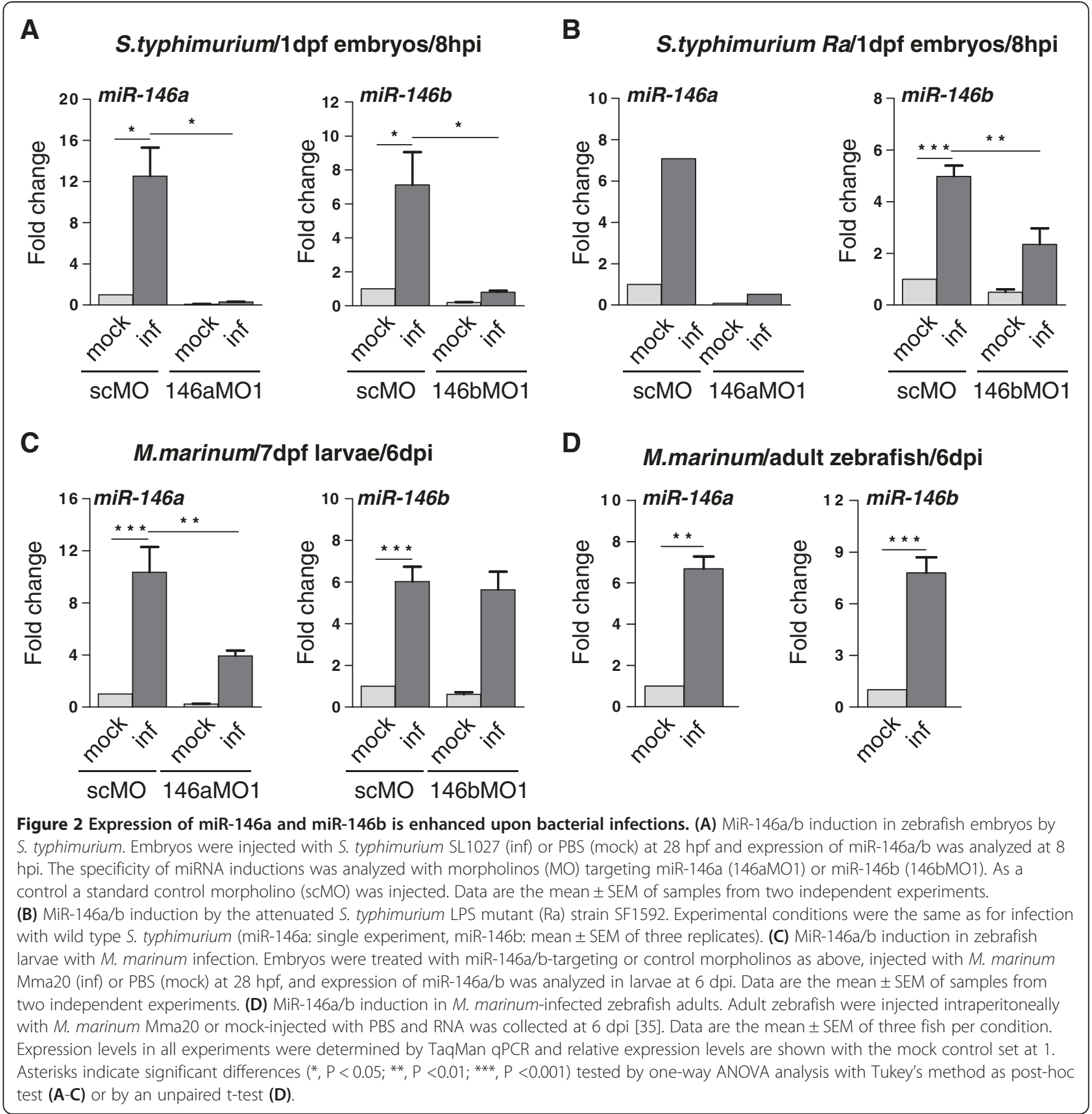

morphants in the numbers of L-plastin-stained leukocytes (Figure 4C) or Mpx-positive neutrophils (Figure 4D) at this stage. Since another study had reported an inhibitory effect of miR-146a knockdown on leukocyte development at 1 $\mathrm{dpf}$ [38], we next analyzed the separate effects of miR-146a and miR-146b knockdown in more detail over a critical period of leukocyte development from 26 to $32 \mathrm{hpf}$. During this period primitive myeloid cells first appear over the yolk sac, and subsequently invade the head. This first wave of primitive myeloid cells is rapidly followed by differentiation of the first erythro-myeloid precursor cells in the caudal blood island region. We performed L-plastin immunostaining with $2 \mathrm{~h}$ intervals over the period from 26 to $32 \mathrm{hpf}$. The total number of L-plastin-positive leukocytes showed a similar increase over this time course between control embryos and embryos injected with 146aMO1, 146aMO2, or $146 \mathrm{bMO} 1$ (Figure 4E-I). 146bMO2 could not be included in this quantitative analysis because it affected embryo development. Nevertheless, L-plastin positive immune cells were still present in 146bMO2 morphants with mild (Figure 4J) or severe phenotypes (Figure 4K). Based on these results, we conclude that miR-146a and miR-146b are 


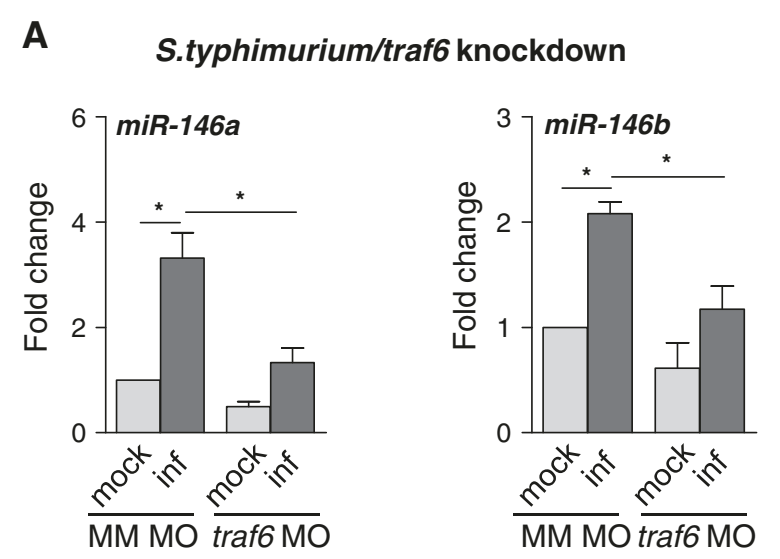

B S.typhimurium /myd88 mutant
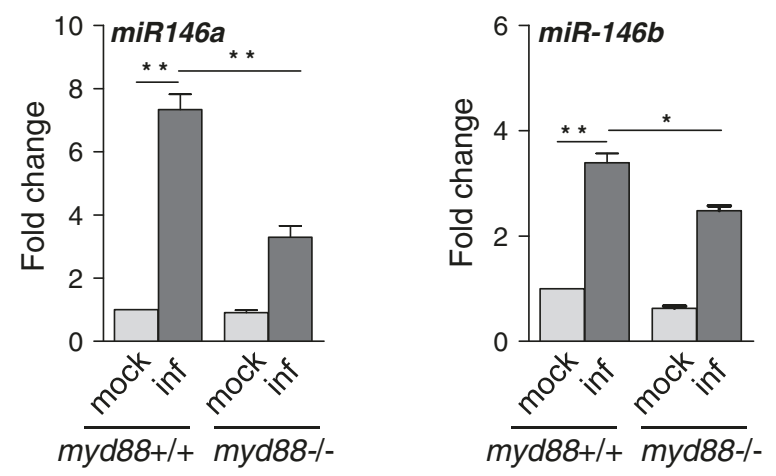

Figure 3 The Traf6-MyD88 pathway is involved in up-regulation of miR-146a and miR-146b expression levels upon infection. (A) Traf6-dependent miR-146a/b induction. Embryos were injected with traf6 morpholino $(\mathrm{MO})$ or a mismatch morpholino (MM) as a control. Embryos were infected at $28 \mathrm{hpf}$ with S. typhimurium (inf) or mock-injected with PBS and samples were collected at 8 hpi. (B) MyD88-dependent miR-146a/b induction. Mutant (myd88-/-) and wild type siblings (myd88+/+) were infected with S. typhimurium at $28 \mathrm{hpf}$ followed by sample collection at 8 hpi. Expression levels in both experiments were determined by TaqMan qPCR and relative expression levels are shown with the mock control set at 1 . Data are the mean \pm SEM of three replicate sample sets. Asterisks indicate significant differences $\left({ }^{*}, P<0.05\right.$; ${ }^{*}$, $P<0.01)$ tested by one-way ANOVA analysis with Tukey's method as post-hoc test.

not required for leukocyte differentiation during zebrafish embryo development.

\section{Combined knockdown of miR-146a and miR-146b does not have a major effect on pro-inflammatory gene expression during $S$. typhimurium infection}

In previous work, we observed that knockdown of a negative regulator of the immune response, the ptpn 6 phosphatase gene, resulted in a hyperinduction of pro-inflammatory gene expression during $S$. typhimurium infection [39]. Since miR-146 has also been proposed as a negative regulator of innate immunity [11], we hypothesized that miR-146 knockdown might have a similar effect. To test this hypothesis, we studied the effect of the combination of miR-146a and miR-146b morpholinos on gene expression in infected and mock-injected embryos by RNA deep sequencing (RNAseq) (Figure 5A). First we analyzed the basal expression differences between morpholino knockdown and the control in mock-injected embryos. Only 68 genes were affected by knockdown of miR-146a and miR-146b, among which 5 genes are members of the p53 signalling pathway (Figure 5B, Additional file 2: Table S1). This might reflect a non-specific effect of the miR-146 knockdown procedure, since morpholino effects on the p53 pathway are relatively common [40]. S. typhimurium infection had a much different effect than morpholino treatment (Figure 5B) and resulted in significant alteration of KEGG pathways related to the immune response and metabolism (Figure 5C), in agreement with previous studies $[34,36,39,41]$. In statistical comparison between uninfected and infected groups, the total number of infection-regulated genes was higher in miR-146 morphants (753 genes) than in control embryos (574 genes) (Figure 5A, Additional file 3: Table S2). Direct statistical comparison between the infected control and morpholino groups also showed a higher number of infection-regulated genes in miR-146 morphants (118 genes, Figure 5A). However, the only pro-inflammatory marker that was induced by infection to higher levels in miR-146 morphants compared with the controls was the matrix metalloproteinase $9(\mathrm{mmp})$ ) gene (Additional file 4: Table S3). RNAseq showed a 1.5-fold higher infection-induction of this gene in miR-146 morphants, which was confirmed by qPCR analysis (Additional file 5: Figure S2). Other pro-inflammatory genes, such as interleukin $1 b(i l 1 b)$, CXCL and CCL family chemokines, and transcriptional regulators of the immune response were induced to similar levels in miR-146 morphants and controls. In conclusion, knockdown of miR-146a and miR-146b in zebrafish embryos did not have a strong effect on innate immunity signalling in the first 8 hours of the response to $S$. typhimurium infection, despite the increased expression of these miRNAs during this phase.

\section{Combined knockdown of miR-146a and miR-146b leads} to increased infection-inducibility of apolipoprotein genes Instead of a significant effect on known innate immune response genes, the RNAseq analysis revealed a possible effect on lipid transport pathways in S. typhimuriuminfected miR-146 morphants. Six members of the apolipoprotein family (Figure $5 \mathrm{C}$ ) were significantly induced upon S. typhimurium infection of miR-146 morphants but not in infected control embryos. To confirm this observation we performed qPCR analysis for the apolipoprotein gene family members (Figure 6). Only $S$. typhimurium-infected embryos were analyzed, since RNAseq analysis (Figure 5) showed an effect of miR-146 morpholinos on apolipoprotein 


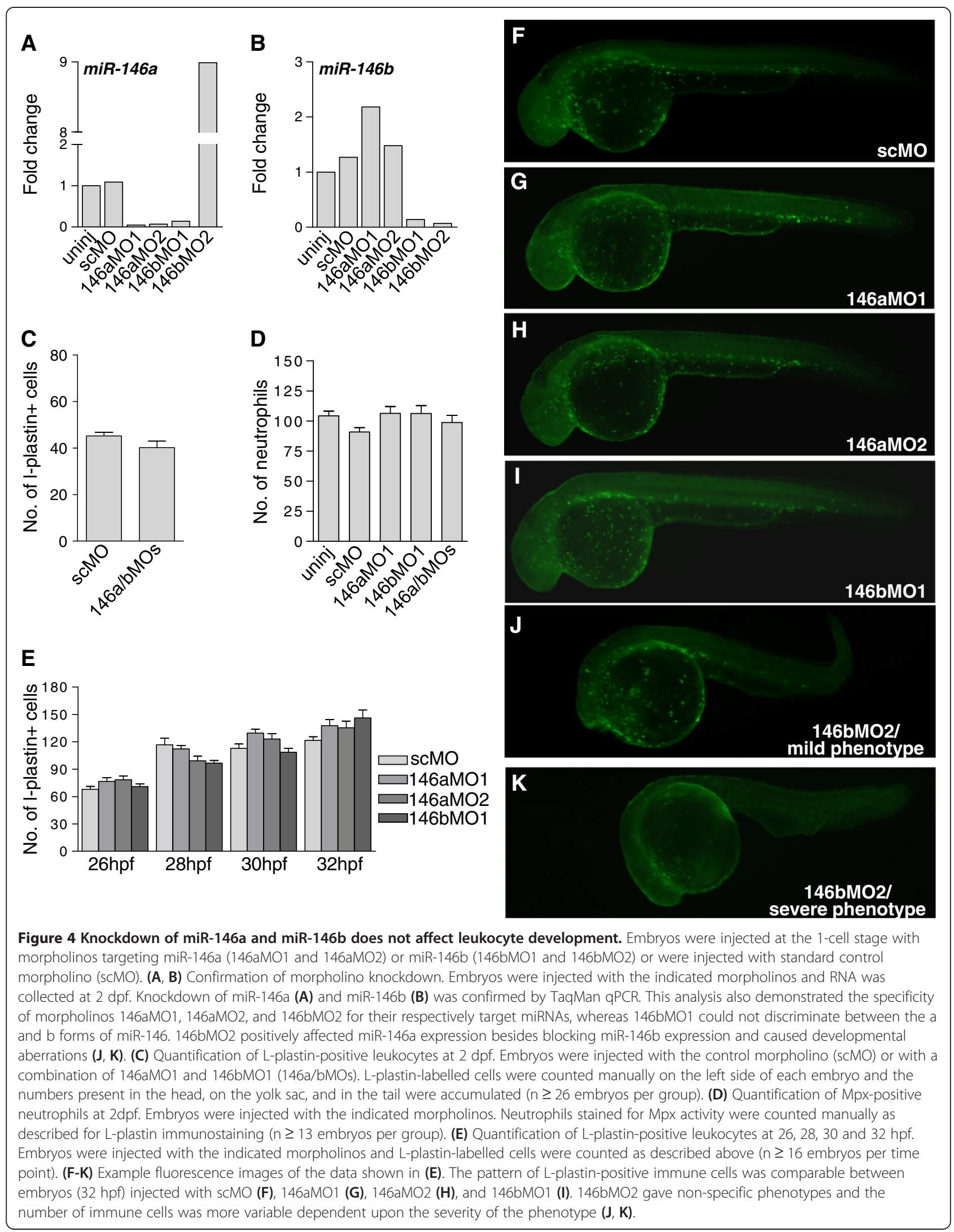




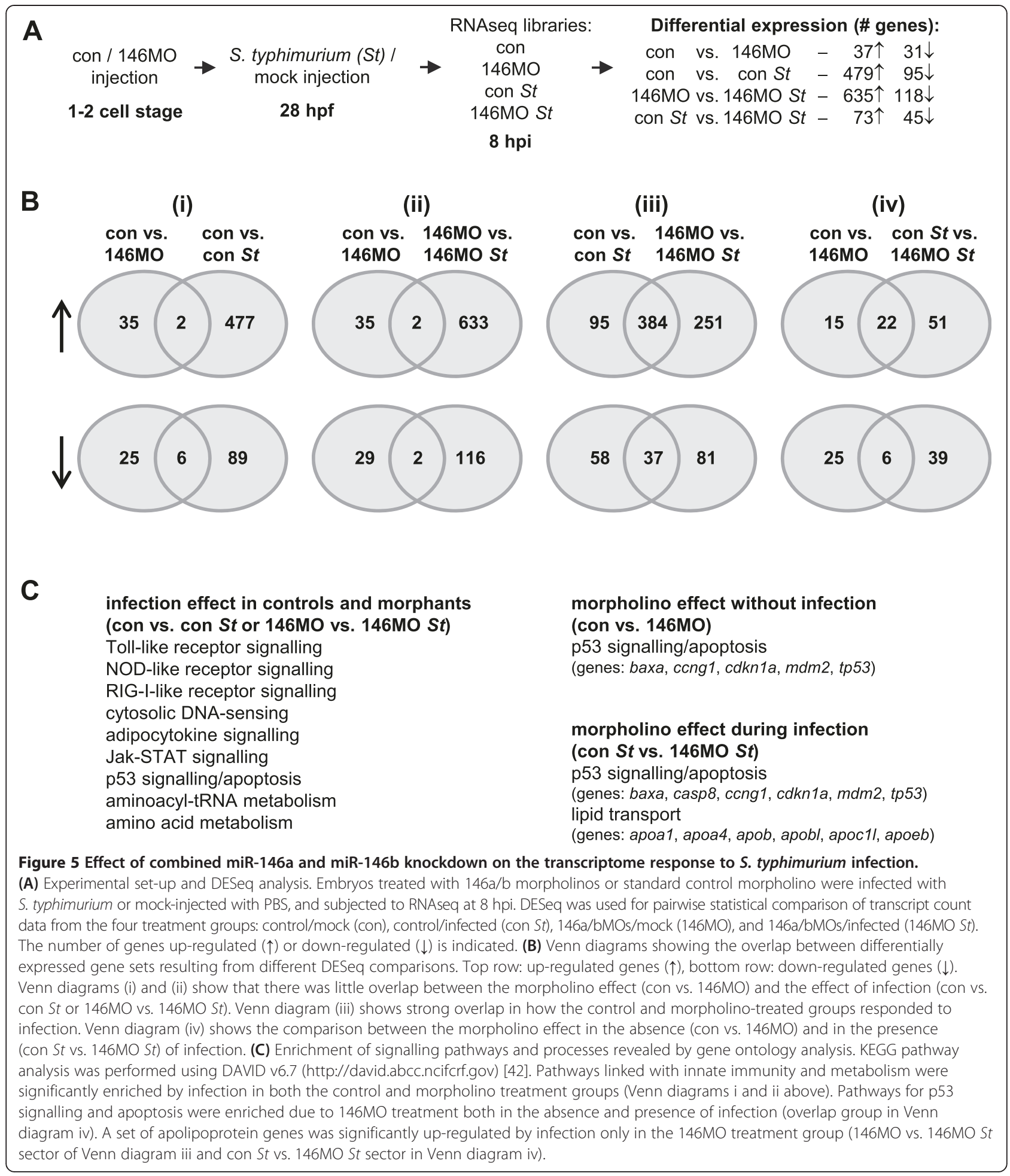

gene expression levels in infected embryos but not in mockinjected controls. Results showed that under conditions of $S$. typhimurium infection, the expression levels of genes apoa1a, apoa4, apoba, apobb, apoc1l, apoeb were between 1.5- and 3- fold higher in miR-146 morphants compared with control embryos. Therefore, miR-146 miRNAs may be involved in fine-tuning of lipid-mediated inflammatory responses of the zebrafish embryo.

\section{Discussion}

By microarray analysis of miRNA expression in zebrafish we found that miRNAs of the miR-21, miR-29, and miR- 


\section{S.typhimurium/1dpf embryos/8hpi}
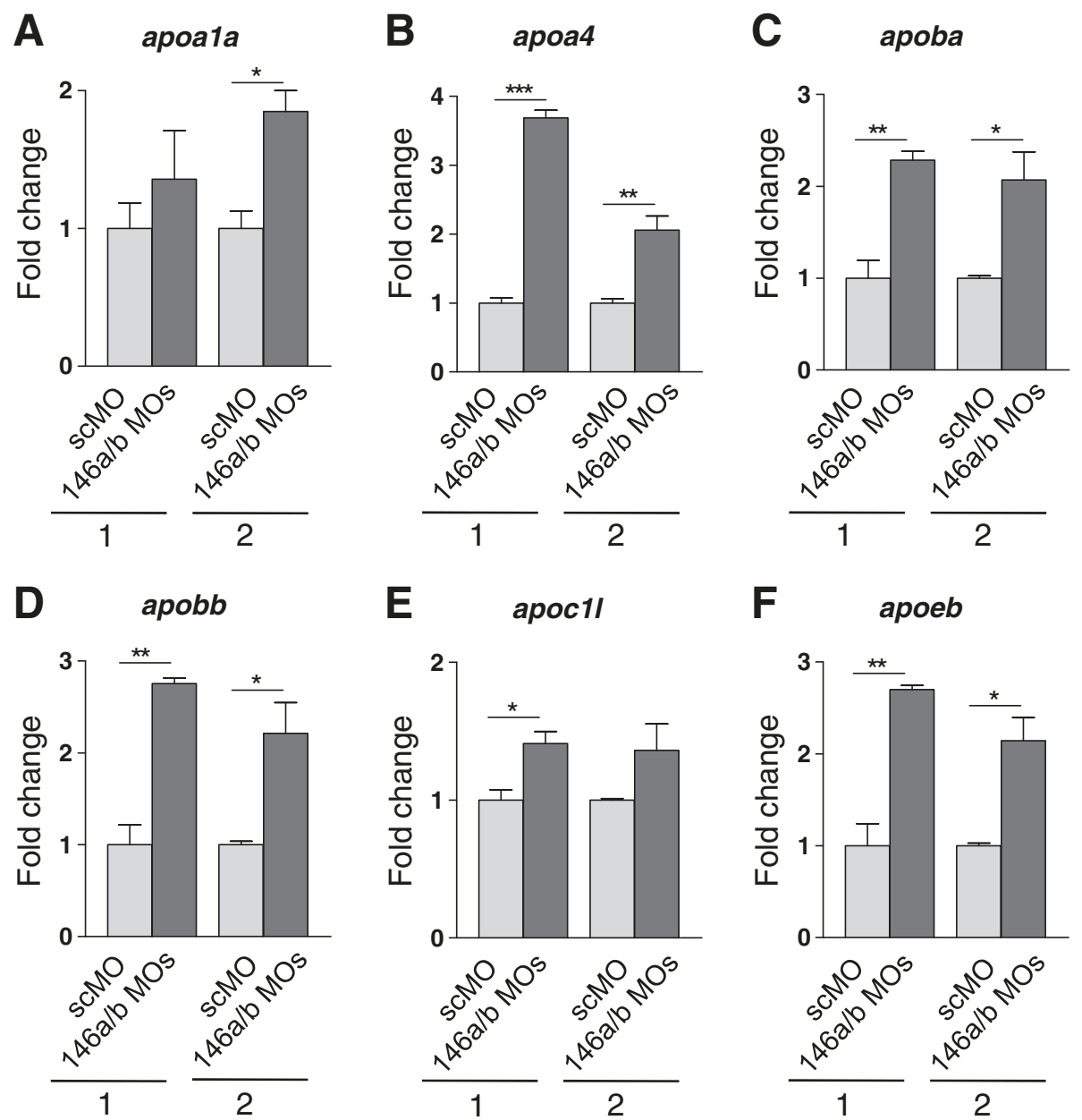

Figure 6 Increased infection-induction of apolipoprotein gene expression under conditions of combined miR-146a and miR-146b knockdown. (A-F) Embryos were injected with control morpholino (scMO) or with a combination of $146 \mathrm{aMO} 1$ and 146bMO1 (146a/bMOs) and infected with S. typhimurium as described in Figure 5. Gene expression levels of six apolipoprotein genes, apoa1 a (A), apoa4 (B), apoba (C), apobb (D), apocll (E), and apoeb (F), were analyzed by qPCR in two replicate infection experiments (1 and 2) and relative expression levels are shown with the lowest expression level set at 1 . Data are the mean \pm SEM of triplicate $\mathrm{qPCR}$ measurements. Asterisks indicate significant differences $\left(*, P<0.05 ;{ }^{* *}, \mathrm{P}<0.01\right)$ determined by an un-paired t-test.

146 families were commonly induced by infection of embryos with S. typhimurium and by infection of adult fish with $M$. marinum. The induction of members of the miR-21, miR-29, and miR-146 families was in line with earlier microarray studies, which reported these along with some other miRNAs, like miR-9, miR-132, miR-147, and miR-155 as infection-inducible [13,26,43,44]. We did not detect altered expression of miR-122 and miR-194, which were found to be inducible during zebrafish infection with Vibrio harveyi [45]. We focused our study on the miR-146 family, which is strongly linked with immune-related diseases in human. We used zebrafish at the embryo stage, when only innate immunity is functional, as an in vivo model to study the role of miR-146 during bacterial infection.
The miR-146a and miR-146b sequences are conserved between zebrafish and human as well as target sites in the 3'-UTR of mRNAs of innate immune pathway genes such as IRAK1 and TRAF6, which are experimentally validated targets of miR-146 [11,26]. Therefore, miR$146 \mathrm{a}$ and miR-146b of zebrafish may function in feedback control of TLR signalling, like the human and murine counterparts $[11,26]$. To study the pathway by which miR-146 expression is induced in zebrafish embryos upon infection we used embryos in which TLR signalling was disrupted by morpholino knockdown of traf6 or by mutation of myd88. The induction levels of miR-146a and miR-146b upon S. typhimurium infection were reduced under conditions of traf6 or myd88 deficiency, but induction was not completely abolished. In the case 
of traf6 deficiency, the residual induction of miRNA expression may be due to a partial morpholino knockdown effect. We have previously shown that mutation of myd88 strongly affects the innate immune response to S. typhimurium infection, but that innate immune genes can still be induced to lower levels in the absence of functional MyD88 [37]. Therefore, it is likely that both the MyD88-Traf6-dependent pathway and parallel MyD88-independent signalling routes contribute to the infection-induced expression of miR-146.

A recent study by Ghani et al. [38] suggested miR$146 \mathrm{a}$ to be required for myeloid cell differentiation in mouse and zebrafish. They reported that miR-146a morpholino knockdown caused an almost complete absence of myeloid cells in zebrafish embryos at $1 \mathrm{dpf}$. However, in our analysis we found no evidence for an inhibitory effect of miR-146 deficiency on myeloid cell development. We used two morpholinos for miR-146a (one of which was the same as a miR-146a morpholino used by Ghani et al. [38]), and verified the knockdown effect by TaqMan qPCR, which showed that the morpholinos effectively inhibited both the basal expression and the infection-induced expression. By immunostaining for the pan-leukocytic marker L-plastin we detected no differences in myeloid cell development between miR-146a morphants and controls over an elaborate time course between 26 and $32 \mathrm{hpf}$, which comprises the critical embryonic stages when myeloid cells differentiate and enter the circulation. Furthermore, no effect on neutrophil differentiation at $2 \mathrm{dpf}$ was detected. For miR-146b we also did not observe an effect on leukocyte development with two different morpholinos targeting this miRNA. Ghani et al. [38] used mRNA in situ hybridization for detection of L-plastin, which might explain the difference with our study in which we used a sensitive immunolabelling method, which is widely used for detection and quantification of myeloid cells in zebrafish embryos [46,47]. Our conclusion that myeloid development in zebrafish embryos is not inhibited by miR-146a or miR$146 \mathrm{~b}$ deficiencies is in line with the phenotype of miR-146a knockout mice, which also were not impaired in myeloid differentiation but in fact showed hyperproliferation of myeloid cells leading to autoimmunity [26].

Knockout mice of miR-146a are hyperresponsive to LPS, showing increased up-regulation of pro-inflammatory cytokines, such as TNF and IL-6 [26]. We used S. typhimurium infection of zebrafish embryos, which results in strong pro-inflammatory gene induction, to analyze the knockdown effect of miR-146a and miR146b by RNAseq analysis. We used a combination of morpholinos against miR-146a and miR-146b in the RNAseq study to avoid that the two miRNAs might compensate for each other's loss-of -function, as their mature sequences differ only by two nucleotides. Several genes in the p53 pathway, including p53 itself, were up-regulated in miR-146 morphants as compared to controls under infected as well as noninfected conditions. This might be attributed to the well known off target effects of morpholino oligonucleotides [40]. However, as miR-146 has been frequently linked with cancer, a direct effect on the p53 pathway cannot be excluded $[18,48,49]$. In fact, one of the p53 pathway genes upregulated by miR-146 knockdown, cdkn1a (p21), is an experimentally validated target of miR-146a in human [50]. In total we found 73 genes which were significantly up-regulated in miR-146 infection as compared to control infection. Besides $c d k n 1 a$, only one other gene, fibrinogen beta chain $(f g b)$, showed an overlap with the predicted targets of zebrafish miR-146a and miR-146b in miRBase. Fibrinogen has roles in cell adhesion, hematopoiesis, and in coagulation and complement cascades associated with primary defence against bacterial infections [51]. Expression levels of other known targets of miR-146 involved in innate immunity, such as irak1, traf6, irf5 and stat1, were not affected, but this was not unexpected since miRNAs can act by translational inhibition.

The matrix metalloproteinase gene $m m p 9$ is one of the most strongly induced pro-inflammatory markers in S. typhimurium infection [34]. The combined morpholino knockdown of miR-146a and miR-146b led to slightly increased induction of this gene during S. typhimurium infection; however, this induction of $m m p 9$ was not accompanied by a general hyperinduction of other proinflammatory markers in the RNAseq analysis. MMP9 is not a predicted target gene of miR-146 in human or zebrafish, but human $M M P 9$ was found to be downregulated upon miR-146a/b overexpression in MDAMB-231 breast cancer cells and in THP-1 macrophages $[20,52]$. This down-regulation was suggested to occur via TLR-mediated and NFKB-dependent pathways rather than by direct targeting of MMP9 [20,52]. Likewise, the induction of zebrafish $m m p 9$ under miR-146a/b knockdown conditions might be an indirect consequence of effects on upstream signalling proteins. In agreement, we have previously shown that $m m p 9$ induction by S. typhimurium infection is mediated by Traf6, which is a known target of miR-146 [11,26,36].

By targeting components of TLR signalling, miR-146 has been shown to function as a negative regulator of the innate immune response in mammals $[11,26]$. However, in our study of $S$. typhimurium infection in zebrafish embryos, miR-146 knockdown did not have a strong impact on the induction of proinflammatory genes. Notably, the effect of miR-146 knockdown was minor in comparison with knockdown analysis of ptpn6, which encodes a SH2domain phosphatase that functions as a negative regulator of innate immunity $[53,54]$. In the same experimental setup, S. typhimurium infection of zebrafish embryos after knockdown of ptpn 6 resulted in hyperinduction of $m m p 9$ 
and a wide range of cytokines, other immune effectors genes, and transcriptional regulators of the immune response [39], while in the case of miR-146 knockdown only mmp9 was slightly higher induced. Furthermore, hyperinflammation in ptpn6 morphants impaired control of S. typhimurium infection [39], while miR-146 knockdown had no such effect (data not shown). These results support that in the early response of zebrafish embryos to S. typhimurium infection Ptpn6 functions as a much stronger negative feedback regulator than miR-146. This would be consistent with the idea that miRNAs function in a subtle fine-tuning of the immune response [1].

While the overall knockdown effect observed in our RNAseq analysis was relatively minor, apolipoproteinmediated lipid transport emerged as an infection-inducible pathway under miR-146 knockdown conditions. Numerous studies have linked apolipoproteins to immunoregulation and host defence [55-57]. MiR-146a has been suggested to be involved in negative regulation of oxidized lowdensity lipoprotein-(LDL) accumulation in macrophages [52]. Lipid accumulation in macrophages is associated with the inflammatory processes that lead to atherosclerosis. The expression of miR-146a was found to be downregulated when THP-1 macrophages were stimulated with oxidized LDL. Furthermore, miR-146 overexpression reduced intracellular LDL cholesterol content and secretion of IL6, IL8, and MMP9 via TLR4-mediated signalling. A similar effect on LDL accumulation was observed by silencing miR-155, another important miRNA regulator of immune processes [58]. Based on its regulatory role in lipid accumulation miR-146a has been proposed as a potential therapeutic candidate for atherosclerosis treatment [52]. Our results support the inhibitory function of miR-146 in lipid-mediated inflammatory responses and the proposed application as a therapeutic target.

\section{Conclusion}

Recent studies have demonstrated the involvement of miRNAs in immune processes and their link to inflammatory disorders and have increased interest to find the molecular pathways responsible for miRNA action [1]. MiR-146 has been recognized as a modulator of the innate and adaptive immune responses in mammals $[11,26,28]$. In a microarray analysis of miRNA expression in zebrafish, both of the miR-146 family members, miR146a and miR-146b, were found to be inducible by S. typhimurium and M. marinum infections. The miR146 family members were commonly induced during infections of embryos and adult fish, along with miRNAs of the miR-21 and miR-29 families, which also have been implicated in immunity and infection. The induction of these miRNAs in embryo infection models links them specifically with the innate immune response, as adaptive immunity is not yet functional at early developmental stages. We exploited the embryo model as an in vivo system to investigate the role of miR-146 in the innate immune response to S. typhimurium infection. Induction of miR-146a and miR-146b by infection was shown to be affected by deficiencies in Traf6 and Myd88, which are central intermediates of Toll-like receptor and cytokine signalling pathways. MiR-146 has previously been implicated in negative feed-back regulation of these pathways $[11,26]$, similar to a number of signalling proteins, including the protein-tyrosine phosphatase Ptpn6 [53,54]. However, whereas knockdown of the ptpn6 gene caused hyperinflammation in zebrafish embryos [39], knockdown of miR-146 in the S. typhimurium embryo infection model had no major effect on pro-inflammatory gene expression or on the expression of transcriptional regulators and signal transduction components of known immune response mediators. In contrast, several members of the apolipoprotein gene family (apoa1a, apoa4, apoba, apobb, apoc1l, and $a p o e b$ ) were infection inducible only under miR-146 knockdown conditions. The apolipoprotein family has been linked to immunoregulation and host defence in many studies $[55,56]$. Consistent with a previous report proposing miR-146a as a negative regulator of LDL accumulation in human macrophages and a therapeutic target for atherosclerosis treatment [52], our results suggest a possible function of miR-146 in regulating apolipoproteinmediated lipid transport under the inflammatory conditions of S. typhimurium infection.

\section{Methods}

\section{Zebrafish husbandry}

Zebrafish were handled in compliance with the local animal welfare regulations and maintained according to standard protocols (zfin.org). The breeding of adult fish was approved by the local animal welfare committee (DEC) of Leiden University. All protocols adhered to the international guidelines specified by the EU Animal Protection Directive 2010/63/EU. Embryos from the zebrafish AB/TL line were used for the infection experiments. In addition, an infection experiment was performed using embryos from a myd88 mutant line and wild type siblings as a control [37]. Embryos were grown at $28-30^{\circ} \mathrm{C}$ in egg water $(60 \mu \mathrm{g} / \mathrm{ml}$ Instant Ocean sea salts). For the duration of bacterial injections embryos were kept under anesthesia in egg water containing $200 \mu \mathrm{g} / \mathrm{mL}$ tricaine (Sigma-Aldrich). Significance cut-offs for DEseq analysis were set at: absolute fold change $\geq 1.5$ and adjusted P-value $\leq 0.1$. Embryos used for immunostaining and Myeloperoxidase (Mpx) assay were kept in egg water containing $0.003 \%$ 1-phenyl-2-thiourea (Sigma-Aldrich) to prevent melanization.

\section{Infection experiments}

S. typhimurium infections were performed using strain SL1027 and its isogenic LPS Ra mutant derivative 
SF1592, carrying the DsRed expression vector, pGMDs3 [59]. M. marinum infections were performed with strain Mma20 expressing mCherry in pSMT3 vector $[35,60]$. Bacteria were grown and prepared for injections as previously described and microinjected into the caudal vein of embryos at 28 hours post fertilization (hpf), using a dose of 200-250 CFUs of S. typhimurium or $100 \mathrm{CFU}$ of $M$. marinum per embryo [61]. As a control, embryos were mock-injected with PBS. After injections, embryos were transferred to fresh egg water and incubated at $28^{\circ} \mathrm{C}$. Adult zebrafish infected with the $M$. marinum Mma20 strain (6 dpi) and PBS-injected control fish were from a previous study [35].

\section{RNA/miRNA isolation and quality check}

Embryos were snap frozen in liquid nitrogen and kept at $-80^{\circ} \mathrm{C}$. Adult zebrafish were homogenized in liquid nitrogen and kept in portions of $50-100 \mu \mathrm{g}$ of powdered tissue at $-80^{\circ} \mathrm{C}$. Total RNA was isolated from frozen embryos or tissue homogenates using the miRNeasy Mini kit (Qiagen) with an on-column DNA purification with RNase Free DNase set (Qiagen). RNA quality of samples for RNAseq analysis was checked with an Agilent Bioanalyzer 2100 using the RNA 6000 Nano series Kit (Agilent, Santa Clara, CA, USA). All samples had a RNA integrity value (RIN) of 10 .

\section{Microarray analysis of miRNAs}

Custom-designed $8 \times 15 \mathrm{k}$ microarray slides were ordered from Agilent Technologies. The $15 \mathrm{k}$ custom design was obtained from Edwin Cuppen and Eugene Berezikov (Hubrecht Institute, Utrecht, The Netherlands) and has been submitted into the Gene Expression Omnibus (GEO) database (GPL 15403). The $15 \mathrm{k}$ design contained a duplicate of 7604 probes of 60-oligonucleotide length. The probes consisted of $2 \times 22$ nucleotide sequences antisense to mature miRNAs separated by a spacer of 8 nucleotides (CGATCTTT) and with a second spacer with the same sequence at the end. From 7604 probes 546 were designed for left $\left(5^{\prime}\right)$ and right ( $\left.3^{\prime}\right)$ arms of the hairpins of zebrafish miRNAs that are known in miRBase, while the remainder 7058 probes corresponded to predicted hairpin structures in the zebrafish genome that might include additional miRNAs. Total RNA, including microRNA, was extracted from pools of 20-30 embryos or from individual adult fish using the miRNeasy Mini $\mathrm{Kit}^{\oplus}$ (Qiagen). Three biological replicates were used for each condition. RNA labelling was carried out with the miRCURY ${ }^{\mathrm{TM}}$ LNA microRNA, Hy ${ }^{\mathrm{TM}} / \mathrm{Hy}^{\mathrm{TM}}$ Power Labelling kit (Exiqon) using $1 \mu \mathrm{g}$ of total RNA according to the manufacturer's instructions. RNA samples from infected embryos or adults were labelled with $\mathrm{Hy} 3$ and hybridized against Hy5-labelled RNA samples from PBS-injected controls. The dual color hybridization of the microarray chips was performed according to Agilent protocol GE2_105_Jan09 (www.Agilent.com) for two-color microarray-based gene expression analysis except that hybridization and washing was performed at $37^{\circ} \mathrm{C}$. The arrays were scanned with DNA Microarray Scanner G2505B from Agilent Technologies. The arrays were scanned twice with 10\% PMT and 100\% PMT laser power. Microarray data was processed from raw data image files with Feature Extraction Software 9.5.3.1 (Agilent Technologies). The XDR function was used to extend the dynamic range. Processed data were subsequently imported into Rosetta Resolver 7.1 (Rosetta Biosoftware, Seattle, Washington) and subjected to default ratio error modelling. Ratio results from control vs. infected replicates were combined using the default ratio experiment builder. Significance cut-offs for the ratios of infected versus control were set at 1.5 -fold change at $\mathrm{P} \leq 10-4$. Significance cut-offs for DEseq analysis were set at: absolute fold change $\geq 1.5$ and adjusted $P$-value $\leq 0.1$. The raw data have been submitted to GEO under accession number GSE45410.

\section{Morpholino knockdown}

Morpholino oligonucleotides (GeneTools) were diluted to the desired concentration in $1 \times$ Danieau buffer (58 mM NaCl, $0.7 \mathrm{mM} \mathrm{KCl,} 0.4 \mathrm{mM} \mathrm{MgSO}_{4}, 0.6 \mathrm{mM}$ $\mathrm{Ca}\left(\mathrm{NO}_{3}\right)_{2}$, $5.0 \mathrm{mM}$ HEPES; $\left.\mathrm{pH} 7.6\right)$ containing $1 \%$ phenol red (Sigma-Aldrich) and approximately $1 \mathrm{nl}$ was injected at the 1-2 cell stage using a Femtojet injector (Eppendorf). For knockdown of miR-146a and miR-146b two morpholinos were used against each of them. The first morpholino for miR-146a (146aMO1: 5'ACCATCTATGGAATTCAGTTCTCAG3') targets the miRNA guide strand and the second morpholino (146aMO2: 5'GAGCCCAUAGAUGAACUUUUCAUGA3') overlaps with the star strand and the dicer cleavage site on the star strand (Additional file 1: Figure S1A). For miR-146b, the first morpholino (146bMO1: 5'GACACCCTTGGAATTCAGTTCTCAA3') also targets the guide strand, and the second morpholino (146bMO2: 5'CGTGGGCTGAATATAAAGCAGACAC3') overlaps with both dicer cleavage sites and part of the star strand (Additional file 1: Figure S1B). All miR-146 morpholinos could be used at a concentration of $0.75 \mathrm{mM}$ without causing morphological defects, except146b-MO2, which was highly toxic. Another morpholino design for miR-146b was not recommended by GeneTools. For traf6 knockdown we used a previously described morpholino [36]. As a control the standard control morpholino (scMO) from GeneTools was used as previously described [39].

\section{Detection of leukocytes}

Embryos were fixed in $4 \%$ paraformaldehyde (PFA) in PBS. Immunofluorescence detection of leukocytes was 
performed with a 1:500 dilution of polyclonal rabbit $\mathrm{Ab}$ against L-plastin [57] and Alexa Fluor 488 goat antirabbit IgG secondary Ab (Molecular Probes), as previously described [62]. Fluorescence images were taken with a Leica MZ16FA stereo fluorescence microscope equipped with a DFC420C digital color camera. Histochemical detection of neutrophils was performed by Mpx activity staining using the Peroxidase Leukocyte Kit (Sigma-Aldrich) as previously described [62].

\section{RNAseq analysis}

For RNAseq analysis, embryos were injected with a combination of 146aMO1 and 146bMO1, or with the scMO. Subsequently, at $28 \mathrm{hpf}$ they were infected with S. typhimurium or mock-injected with PBS, and RNA was isolated from pools of at least 50 embryos at 8 hours post injection (hpi). Two independent experiments were performed for RNAseq analysis of biological duplicates. A total of $3 \mu \mathrm{g}$ of RNA was used to make RNAseq libraries using the Illumina TruSeq RNA Sample Preparation Kit v2 (Illumina Inc., San Diego, USA). In the manufacturer's instructions two modifications were made. In the adapter ligation step $1 \mu \mathrm{l}$ instead of $2.5 \mu \mathrm{l}$ adapter was used. In the library size selection step the library fragments were isolated with a double Ampure XP purification with a $0.7 \times$ beads to library ratio. The resulting mRNA-Seq library was sequenced using an Illumina HiSeq2000 instrument according to the manufacturer's description with a read length of $2 \times 50$ nucleotides. Image analysis and base calling was done by the Illumina HCS version 1.15.1. Sequence reads were quality trimmed using the quality_trim module in the CLCbio Assembly Cell v4.0.6. Filtered reads were mapped to Ensembl transcripts (Zv9_63) using the ref_assemble_short module in the CLCbio Assembly Cell v4.0.6. Accumulation of transcripts to Ensembl genes was done by first converting the mapping files to a table with the assembly_table module in the CLCbio Assembly Cell v4.0.6. Secondly a custom script was used that sums all reads belonging to a transcript. Non-uniquely mapped reads were divided between transcripts according to their ratio of uniquely mapped reads. Finally, read counts of transcripts belonging to the same gene were summed to obtain count data at Ensembl gene level. Foldchange and differential expression significance values were calculated from gene level read counts using the DESeq package (version 1.8.3) available in Bioconductor (version 2.10). DESeq utilizes a negative binomial distribution for modeling read counts per gene and implements a method for normalizing the counts [63]. Significance cut-offs for DEseq analysis were set at: absolute fold change $\geq 1.5$ and adjusted Pvalue $\leq 0.1$. The raw data have been submitted to GEO under accession number GSE45410.

\section{Quantitative RT-PCR analysis}

For quantification of miR-146a/b expression levels, RTPCR reactions were performed using a TaqMan microRNA reverse transcription kit (Applied Biosystem) according to the manufacturer's instructions. Briefly, 10 ng of total RNA was reverse transcribed using $5 \times \mathrm{RT}$ primers (Custom TaqMan small RNA Assay-Applied Biosystem) in a total reaction volume of $15 \mu \mathrm{l}$. Reactions were kept on ice for $5 \mathrm{~min}$ and then were transferred to the thermal cycler for incubations at $16^{\circ} \mathrm{C}$ and $42^{\circ} \mathrm{C}$ for $30 \mathrm{~min}$ each, followed by an incubation at $85^{\circ} \mathrm{C}$ for $5 \mathrm{~min}$. Quantitative RT-PCR with $0.665 \mu \mathrm{l}$ cDNA input per reaction was performed using a Custom TaqMan small RNA Assay for each miRNA and a TaqMan Universal PCR Master Mix (Applied Biosystem) in a total of $10 \mu \mathrm{l}$ per reaction. Cycle threshold values were calculated under the parameters of 40 cycles of $10 \mathrm{~min}$ at $95^{\circ} \mathrm{C}, 15 \mathrm{sec}$ at $95^{\circ} \mathrm{C}$ and $60 \mathrm{sec}$ at $60^{\circ} \mathrm{C}$. All reactions were performed in triplicate. For normalization, miR-222, which showed no changes in response to bacterial challenge, was taken as reference. Results were analyzed using the $\Delta \Delta \mathrm{Ct}$ method. Quantification of $m m p 9$ and apolipoprotein gene expression was performed as previously described [39], using the ppial gene for normalization and the primers in Additional file 6: Table S4.

\section{Availability of supporting data}

The data sets supporting the results of this article are available in the Gene Expression Omnibus repository under accession number GSE45410 (http://www.ncbi. nlm.nih.gov/geo/query/acc.cgi?acc=GSE45410).

\section{Additional files}

\begin{abstract}
Additional file 1: Figure S1. Target sites of miR-146a and miR-146b morpholinos on their respective miRNAs. The stem-loop sequences of the zebrafish miR-146a and miR-146b homologs, dre-miR-146a (A) and dre-miR-146b (B) are shown with the miRNA guide strand in lower case The activity of miRNAs can be blocked using morpholinos complementary to the miRNA guide strand or to the Drosha or Dicer nucleolytic processing sites of the primary miRNA or pre-miRNA (www. gene-tools.com). Regions targeted by the morpholinos are indicated in blue (146aMO1 and 146bMO1) or yellow (146aMO2 and 146bMO2), and overlap between two morpholino regions is shown in green.
\end{abstract}

Additional file 2: Table S1. Effect of miR-146a and miR-146b knockdown on gene expression in the absence of infection. The table lists the genes showing significantly up- or down-regulated expression under knockdown conditions of miR-146a and miR-146b in comparison with control embryos (experimental set-up shown in Figure 5A). Significance cut-offs were: $-1.5 \leq$ fold change $\leq 1.5$ and adjusted P-value $\leq 0.1$ in statistical analysis with DESeq. Some genes were undetectable in one of the samples, resulting in an infinite fold change, which is indicated in the table as Inf or Inf down for up- and down-regulated genes, respectively. Genes are annotated by Ensembl ID, description and gene symbol.

Additional file 3: Table S2. Effect of S. typhimurium infection on gene expression in control embryos and under conditions of miR-146a and miR-146b knockdown. The table lists the genes showing significantly up- or down-regulated expression due to S. typhimurium infection in embryos injected with a standard control morpholino or with a 
combination of morpholinos targeting miR-146a and miR-146b (experimental set-up shown in Figure $5 A$ ). Significance cut-offs were: $-1.5 \leq$ fold change $\leq 1.5$ and adjusted P-value $\leq 0.1$ in statistical analysis with DESeq. Significant values are marked yellow. Some genes were undetectable in one of the samples, resulting in an infinite fold change, which is indicated in the table as Inf or Inf down for up- and down-regulated genes respectively. Genes are annotated by Ensembl ID, description and gene symbol.

Additional file 4: Table S3. Effect of miR-146a and miR-146b knockdown on gene expression during S. typhimurium infection. The table lists the genes showing significantly up- or down-regulated expression in S. typhimurium-infected embryos under knockdown conditions of miR-146a and miR-146b in comparison with S. typhimurium-infected control embryos (experimental set-up shown in Figure 5A). Significance cut-offs were: $-1.5 \leq$ fold change $\leq 1.5$ and adjusted P-value $\leq 0.1$ in statistical analysis with DESeq. Some genes were undetectable in one of the samples, resulting in an infinite fold change, which is indicated in the table as Inf or Inf down for up- and down-regulated genes, respectively. Genes are annotated by Ensembl ID, description and gene symbol.

Additional file 5: Figure S2. Increased $m m p 9$ expression in S. typhimurium infection under knockdown conditions of miR-146a and miR-146b. Embryos were injected with control morpholino (scMO) or with a combination of 146aMO1 and 146bMO1 (146a/bMOs) and infected with S. typhimurium or mock-injected with PBS as described in Figure 5. Gene expression of $m m p 9$ was analyzed by $\mathrm{qPCR}$ and relative expression levels are shown with the mock control set at 1. The $\mathrm{mmp} 9$ induction level after $S$. typhimurium infection was significantly higher in miR-146a/miR-146b morphants than in control embryos, consistent with the results of RNAseq analysis (Additional file 4: Table S3). Data are the mean \pm SEM of samples from two independent experiments. Asterisks indicate significant differences $\left({ }^{*}, \mathrm{P}<0.05 ;{ }^{* *}, \mathrm{P}<0.01\right.$ ) tested by one-way ANOVA analysis with Tukey's method as post-hoc test.

Additional file 6: Table S4. Primer sequences for qPCR analysis of apolipoprotein genes. Forward and reverse primer sequences for apolipoprotein genes $\mathrm{A}-1 \mathrm{a}, \mathrm{A}-\mathrm{IV}, \mathrm{Ba}, \mathrm{Bb}, \mathrm{C} 1$-like, and Eb.

\section{Competing interests}

The authors declare that they have no competing interests.

\section{Authors' contributions}

$\mathrm{AO}$ and ZK performed experiments, analyzed data, prepared figures and wrote the manuscript. VL performed experiments and analyzed data. JJYR analyzed data. MM and HPS provided materials and resources for this study and gave input for the manuscript. AHM conceived and supervised the study and wrote the manuscript. All authors read and approved the final version.

\section{Acknowledgements}

The authors thank Eugene Berezikov and Edwin Cuppen (Hubrecht Institute, Utrecht, The Netherlands) for the Agilent miRNA array design and Hans Jansen (ZF-screens, Leiden, The Netherlands) for help with RNAseq analysis. This work was supported by the European Commission 6th Framework Project ZF-TOOLS (LSHG-CT-2006-037220) and by the Smart Mix Program of The Netherlands Ministry of Economic Affairs and the Ministry of Education, Culture and Science. Additionally, JJYR was supported by Marie Curie fellowship of the European 7th Framework Initial Training Network FishForPharma (PITG-GA-2011-289209) and ZK by a fellowship of the Higher Education Committee of Pakistan.

\section{Author details}

${ }^{1}$ Institute of Biology, Leiden University, Einsteinweg 55, 2333 CC Leiden, The Netherlands. ${ }^{2}$ Department of Genetics, Faculty of Science and Informatics, University of Szeged, 52 Kozepfasor, H-6726 Szeged, Hungary.

Received: 19 June 2013 Accepted: 17 September 2013

Published: 10 October 2013

\section{References}

1. O'Neill LA, Sheedy FJ, McCoy CE: MicroRNAs: the fine-tuners of toll-like receptor signalling. Nat Rev Immunol 2011, 11(3):163-175.

2. Kasinski AL, Slack FJ: Epigenetics and genetics. MicroRNAs en route to the clinic: progress in validating and targeting microRNAs for cancer therapy. Nat Rev Cancer 2011, 11(12):849-864.

3. Ceribelli A, Satoh M, Chan EK: MicroRNAs and autoimmunity. Curr Opin Immunol 2012, 24(6):686-691.

4. Iborra $M$, Bernuzzi $F$, Invernizzi $P$, Danese $S$ : MicroRNAs in autoimmunity and inflammatory bowel disease: crucial regulators in immune response. Autoimmun Rev 2012, 11(5):305-314.

5. Foster PS, Plank M, Collison A, Tay HL, Kaiko GE, Li J, Johnston SL, Hansbro PM, Kumar RK, Yang $M$, et al: The emerging role of microRNAs in regulating immune and inflammatory responses in the lung. Immunol Rev 2013, 253(1):198-215.

6. Huntzinger $E$, lzaurralde $E$ : Gene silencing by microRNAs: contributions of translational repression and mRNA decay. Nat Rev Genet 2011, 12(2):99-110

7. O'Connell RM, Rao DS, Chaudhuri AA, Baltimore D: Physiological and pathological roles for microRNAs in the immune system. Nat Rev Immunol 2010, 10(2):111-122.

8. Pauli A, Rinn JL, Schier AF: Non-coding RNAs as regulators of embryogenesis. Nat Rev Genet 2011, 12(2):136-149.

9. Baltimore D, Boldin MP, O'Connell RM, Rao DS, Taganov KD: MicroRNAs: new regulators of immune cell development and function. Nat Immunol 2008, 9(8):839-845.

10. Lindsay MA: microRNAs and the immune response. Trends Immunol 2008, 29(7):343-351.

11. Taganov KD, Boldin MP, Chang KJ, Baltimore D: NF-kappaB-dependent induction of microRNA miR-146, an inhibitor targeted to signaling proteins of innate immune responses. Proc Natl Acad Sci U S A 2006, 103(33):12481-12486.

12. Tili E, Michaille JJ, Cimino A, Costinean S, Dumitru CD, Adair B, Fabbri M Alder H, Liu CG, Calin GA, et al: Modulation of miR-155 and miR-125b levels following lipopolysaccharide/TNF-alpha stimulation and their possible roles in regulating the response to endotoxin shock. $J$ Immunol 2007, 179(8):5082-5089.

13. Liu G, Friggeri A, Yang Y, Park YJ, Tsuruta Y, Abraham E: miR-147, a microRNA that is induced upon toll-like receptor stimulation, regulates murine macrophage inflammatory responses. Proc Natl Acad Sci U S A 2009, 106(37):15819-15824.

14. Rodriguez A, Vigorito E, Clare S, Warren MV, Couttet P, Soond DR, van Dongen S, Grocock RJ, Das PP, Miska EA, et al: Requirement of bic/ microRNA-155 for normal immune function. Science 2007 316(5824):608-611.

15. Schulte LN, Eulalio A, Mollenkopf HJ, Reinhardt R, Vogel J: Analysis of the host microRNA response to salmonella uncovers the control of major cytokines by the let-7 family. EMBO J 2011, 30(10):1977-1989.

16. Liu Y, Chen Q, Song Y, Lai L, Wang J, Yu H, Cao X, Wang Q: MicroRNA-98 negatively regulates IL-10 production and endotoxin tolerance in macrophages after LPS stimulation. FEBS Lett 2011, 585(12):1963-1968.

17. Ma F, Xu S, Liu X, Zhang O, Xu X, Liu M, Hua M, Li N, Yao H, Cao X: The microRNA miR-29 controls innate and adaptive immune responses to intracellular bacterial infection by targeting interferon-gamma. Nat Immunol 2011, 12(9):861-869.

18. Labbaye C, Testa U: The emerging role of MIR-146A in the control of hematopoiesis, immune function and cancer. J Hematol Oncol 2012, 5:13.

19. Rusca N, Monticelli S: MiR-146a in immunity and disease. Mol Biol Int 2011, 2011:437301.

20. Bhaumik D, Scott GK, Schokrpur S, Patil CK, Campisi J, Benz CC: Expression of microRNA-146 suppresses NF-kappaB activity with reduction of metastatic potential in breast cancer cells. Oncogene 2008, 27(42):5643-5647.

21. Hurst DR, Edmonds MD, Scott GK, Benz CC, Vaidya KS, Welch DR: Breast cancer metastasis suppressor 1 up-regulates miR-146, which suppresses breast cancer metastasis. Cancer Res 2009, 69(4):1279-1283.

22. Xia H, Qi Y, Ng SS, Chen X, Li D, Chen S, Ge R, Jiang S, Li G, Chen Y, et al: microRNA-146b inhibits glioma cell migration and invasion by targeting MMPs. Brain Res 2009, 1269:158-165.

23. Yao Q, Cao Z, Tu C, Zhao Y, Liu H, Zhang S: MicroRNA-146a acts as a metastasis suppressor in gastric cancer by targeting WASF2. Cancer Lett 2013, 335(1):219-224. 
24. Xu WD, Lu MM, Pan HF, Ye DQ: Association of MicroRNA-146a with autoimmune diseases. Inflammation 2012, 35(4):1525-1529.

25. Chan EK, Ceribelli A, Satoh M: MicroRNA-146a in autoimmunity and innate immune responses. Ann Rheum Dis 2013, 72(Suppl 2):ii90-ii95.

26. Boldin MP, Taganov KD, Rao DS, Yang L, Zhao JL, Kalwani M, Garcia-Flores Y, Luong M, Devrekanli A, Xu J, et al: miR-146a is a significant brake on autoimmunity, myeloproliferation, and cancer in mice. J Exp Med 2011, 208(6):1189-1201.

27. Tang Y, Luo X, Cui H, Ni X, Yuan M, Guo Y, Huang X, Zhou H, de Vries N, Tak PP, et al: MicroRNA-146A contributes to abnormal activation of the type I interferon pathway in human lupus by targeting the key signaling proteins. Arthritis Rheum 2009, 60(4):1065-1075.

28. Yang L, Boldin MP, Yu Y, Liu CS, Ea CK, Ramakrishnan P, Taganov KD, Zhao JL, Baltimore D: miR-146a controls the resolution of $T$ cell responses in mice. J Exp Med 2012, 209(9):1655-1670.

29. Meijer AH, Spaink HP: Host-pathogen interactions made transparent with the zebrafish model. Curr Drug Targets 2011, 12(7):1000-1017.

30. Renshaw SA, Trede NS: A model 450 million years in the making: zebrafish and vertebrate immunity. Dis Model Mech 2012, 5(1):38-47.

31. van der Vaart M, Spaink HP, Meijer AH: Pathogen recognition and activation of the innate immune response in zebrafish. Adv Hemato 2012, 2012:159807.

32. Chen PY, Manninga H, Slanchev K, Chien M, Russo JJ, Ju J, Sheridan R, John B, Marks DS, Gaidatzis D, et al: The developmental miRNA profiles of zebrafish as determined by small RNA cloning. Genes Dev 2005, 19(11):1288-1293

33. Soares AR, Pereira PM, Santos B, Egas C, Gomes AC, Arrais J, Oliveira JL, Moura GR, Santos MA: Parallel DNA pyrosequencing unveils new zebrafish microRNAs. BMC Genomics 2009, 10:195.

34. Stockhammer OW, Zakrzewska A, Hegedus Z, Spaink HP, Meijer AH: Transcriptome profiling and functional analyses of the zebrafish embryonic innate immune response to salmonella infection. J Immunol 2009, 182(9):5641-5653.

35. van der Sar AM, Spaink HP, Zakrzewska A, Bitter W, Meijer AH: Specificity of the zebrafish host transcriptome response to acute and chronic mycobacterial infection and the role of innate and adaptive immune components. Mol Immunol 2009, 46(11-12):2317-2332.

36. Stockhammer OW, Rauwerda H, Wittink FR, Breit TM, Meijer AH, Spaink HP: Transcriptome analysis of Traf6 function in the innate immune response of zebrafish embryos. Mol Immunol 2010, 48(1-3):179-190.

37. van der Vaart M, van Soest JJ, Spaink HP, Meijer AH: Functional analysis of a zebrafish myd88 mutant identifies key transcriptional components of the innate immune system. Dis Model Mech 2013, 6(3):841-854

38. Ghani S, Riemke P, Schonheit J, Lenze D, Stumm J, Hoogenkamp M, Lagendijk A, Heinz S, Bonifer C, Bakkers J, et al: Macrophage development from HSCs requires PU.1-coordinated microRNA expression. Blood 2011, 118(8):2275-2284

39. Kanwal Z, Zakrzewska A, den Hertog J, Spaink HP, Schaaf MJ, Meijer AH: Deficiency in hematopoietic phosphatase ptpn6/Shp1 hyperactivates the innate immune system and impairs control of bacterial infections in zebrafish embryos. J Immunol 2013, 190(4):1631-1645.

40. Robu ME, Larson JD, Nasevicius A, Beiraghi S, Brenner C, Farber SA, Ekker SC: p53 activation by knockdown technologies. PLoS Genet 2007, 3(5):e78.

41. Ordas A, Hegedus Z, Henkel CV, Stockhammer OW, Butler D, Jansen HJ, Racz P, Mink M, Spaink HP, Meijer AH: Deep sequencing of the innate immune transcriptomic response of zebrafish embryos to Salmonella infection. Fish Shellfish Immunol 2011, 31(5):716-724

42. Huang DW, Sherman BT, Lempicki RA: Systematic and integrative analysis of large gene lists using DAVID bioinformatics resources. Nat Protoc 2009, 4(1):44-57.

43. Bazzoni F, Rossato M, Fabbri M, Gaudiosi D, Mirolo M, Mori L, Tamassia N, Mantovani A, Cassatella MA, Locati M: Induction and regulatory function of miR-9 in human monocytes and neutrophils exposed to proinflammatory signals. Proc Natl Acad Sci U S A 2009, 106(13):5282-5287.

44. Sheedy FJ, Palsson-McDermott E, Hennessy EJ, Martin C, O'Leary JJ, Ruan QG, Johnson DS, Chen YH, O'Neill LAJ: Negative regulation of TLR4 via targeting of the proinflammatory tumor suppressor PDCD4 by the microRNA miR-21. Nat Immunol 2010, 11(2):141-U159.

45. Wu TH, Pan CY, Lin MC, Hsieh JC, Hui CF, Chen JY: In vivo screening of zebrafish microRNA responses to bacterial infection and their possible roles in regulating immune response genes after lipopolysaccharide stimulation. Fish Physiol Biochem 2012, 38(5):1299-1310.

46. Mathias JR, Dodd ME, Walters KB, Rhodes J, Kanki JP, Look AT, Huttenlocher A: Live imaging of chronic inflammation caused by mutation of zebrafish Hai1. J Cell Sci 2007, 120(19):3372-3383.

47. Feng $Y$, Santoriello C, Mione M, Hurlstone A, Martin P: Live imaging of innate immune cell sensing of transformed cells in zebrafish larvae: parallels between tumor initiation and wound inflammation. Plos Biol 2010, 8(12):e1000562

48. Sassen S, Miska EA, Caldas C: MicroRNA - implications for cancer. Virchows Arch 2008, 452(1):1-10.

49. Visone R, Croce CM: MiRNAs and cancer. Am J Pathol 2009, 174(4):1131-1138.

50. Borgdorff $V$, Lleonart ME, Bishop CL, Fessart D, Bergin AH, Overhoff MG, Beach DH: Multiple microRNAs rescue from Ras-induced senescence by inhibiting p21(Waf1/Cip1). Oncogene 2010, 29(15):2262-2271.

51. Rivera J, Vannakambadi G, Hook M, Speziale P: Fibrinogen-binding proteins of Gram-positive bacteria. Thromb Haemostasis 2007, 98(3):503-511.

52. Yang K, He YS, Wang XQ, Lu L, Chen QJ, Liu J, Sun Z, Shen WF: MiR-146a inhibits oxidized low-density lipoprotein-induced lipid accumulation and inflammatory response via targeting toll-like receptor 4. Febs Letters 2011, 585(6):854-860.

53. An HZ, Hou J, Zhou J, Zhao W, Xu HM, Zheng YJ, Yu YZ, Liu SX, Cao XT: Phosphatase SHP-1 promotes TLR- and RIG-I-activated production of type I interferon by inhibiting the kinase IRAK1. Nat Immunol 2008, 9(5):542-550.

54. Croker BA, Lawson BR, Rutschmann S, Berger M, Eidenschenk C, Blasius AL, Moresco EMY, Sovath S, Cengia L, Shultz LD, et al: Inflammation and autoimmunity caused by a SHP1 mutation depend on IL-1, MyD88, and a microbial trigger (vol 105, pg 15028, 2008). Proc Natl Acad Sci U S A 2008, 105(49):19561-19561.

55. Khovidhunkit W, Kim MS, Memon RA, Shigenaga JK, Moser AH, Feingold KR, Grunfeld C: Effects of infection and inflammation on lipid and lipoprotein metabolism: mechanisms and consequences to the host. J Lipid Res 2004, 45(7):1169-1196

56. Li L, Thompson PA, Kitchens RL: Infection induces a positive acute phase apolipoprotein $\mathrm{E}$ response from a negative acute phase gene: role of hepatic LDL receptors. J Lipid Res 2008, 49(8):1782-1793.

57. Raffai RL: Apolipoprotein E regulation of myeloid cell plasticity in atherosclerosis. Curr Opin Lipidol 2012, 23(5):471-478.

58. Huang RS, Hu GQ, Lin B, Lin ZY, Sun CC: MicroRNA-155 Silencing enhances inflammatory response and lipid uptake in oxidized lowdensity lipoprotein-stimulated human THP-1 macrophages. J Invest Med 2010, 58(8):961-967.

59. van der Sar AM, Musters RJP, van Eeden FJM, Appelmelk B」, Vandenbroucke-Grauls CMJE, Bitter W: Zebrafish embryos as a model host for the real time analysis of Salmonella typhimurium infections. Cell Microbiol 2003, 5(9):601-611.

60. van der Sar AM, Abdallah AM, Sparrius M, Reinders E, Vandenbroucke-Grauls CMJE, Bitter W: Mycobacterium marinum strains can be divided into two distinct types based on genetic diversity and virulence. Infect Immun 2004, 72(11):6306-6312.

61. Benard EL, van der Sar AM, Ellett F, Lieschke GJ, Spaink HP, Meijer AH: Infection of zebrafish embryos with intracellular bacterial pathogens. J Vis Exp 2012, 61:e3781.

62. Cui C, Benard EL, Kanwal Z, Stockhammer OW, van der Vaart M, Zakrzewska A, Spaink HP, Meijer AH: Infectious disease modeling and innate immune function in zebrafish embryos. Methods Cell Biol 2011, 105:273-308.

63. Anders S, Huber W: Differential expression analysis for sequence count data. Genome Biol 2010, 11(10):R106.

\section{doi:10.1186/1471-2164-14-696}

Cite this article as: Ordas et al: MicroRNA-146 function in the innate immune transcriptome response of zebrafish embryos to Salmonella typhimurium infection. BMC Genomics 2013 14:696. 